\title{
A single-stress model for the prediction of yielding of unsaturated cemented soils under isotropic loads
}

\author{
Agostino Walter Bruno ${ }^{1, *}$, Domenico Gallipoli ${ }^{2}$, Mohamed Rouainia $^{1}$, and Marti Lloret-Cabot ${ }^{3}$ \\ ${ }^{1}$ School of Engineering, Geotechnics and Structures, Newcastle University, NE1 7RU Newcastle upon Tyne, United Kingdom \\ ${ }^{2}$ Laboratoire SIAME, Fédération IPRA, Université de Pau et des Pays de l'Adour, 64600 Anglet, France \\ ${ }^{3}$ Department of Engineering, Durham University, DH1 3LE Durham, United Kingdom
}

\begin{abstract}
This paper presents a bounding surface model predicting the combined effects of cementation and partial saturation on the mechanical behaviour of soils subjected to isotropic loading. The loss of cementation caused by loading, wetting or drying of a normally consolidated soil is described by a "cementation bonding function". This states that, under virgin conditions, the ratio between cemented and uncemented void ratios monotonically decreases with increasing levels of scaled stress. The scaled stress is the variable governing the intrinsic behaviour of the soil under both saturated and unsaturated conditions. Combination of the cementation bounding function with a previously proposed model for unsaturated soil behaviour leads to the formulation of a "cemented unified normal compression line" (CUNCL). This describes the virgin behaviour of both cemented and uncemented soils under saturated and unsaturated conditions. Progressive yielding is modelled by assuming that the slope of the generic loading curve tends towards the slope of the CUNCL as the soil state moves from overconsolidated to virgin conditions. The model has been calibrated and validated against existing experimental data demonstrating a good ability to predict the void ratio of cemented soils during isotropic loading, unloading and wetting under both saturated and unsaturated conditions.
\end{abstract}

\section{Introduction}

Soils are often found in nature in a cemented state and under partially saturated conditions. The evolution of both cementation and partial saturation during loading, wetting or drying paths strongly affects the mechanical behaviour of soils. On one hand, cementation is progressively damaged as the applied loads increase and the interparticle linkages are destroyed. On the other, rainfall infiltration and evapotranspiration, change the degree of saturation of the soil, thus modifying the capillary bonds between particles.

Over the last three decades, researchers have developed constitutive laws that predict the gradual loss of soil cementation under increasing loads [1-11]. However, these models neglect the effect of partial saturation on the mechanical behaviour of cemented soils. Other authors have instead devised mechanical models that predict the unsaturated behaviour without accounting for the effect of cementation [12-20]. Only a handful of studies have proposed elastoplastic models that take into account the combined effect of cementation and partial saturation [2126]. These models adopt distinct sets of equations to describe the soil behaviour in the elastic and elasto-plastic regimes by introducing yielding surfaces and hardening rules that govern the transition between the two regimes. At each stress increment, it is therefore necessary to establish whether the soil is behaving elastically or elastoplastically in order to use the right set of equations, which increases computational demands.

To overcome these limitations, this paper proposes a bounding surface model that predicts the gradual yielding of unsaturated cemented soils subjected to isotropic loads without any discontinuity between elastic and elastoplastic states. The formulation is also able to predict the smooth variation of void ratio during loading-unloading cycles.

The model originates from the definition of a cementation bonding function, which describes the progressive loss of porosity sustained by cementation as the scaled stress increases under virgin conditions, and the inter-particle linkages are therefore destroyed. The scaled stress is the constitutive variable governing the behaviour of the uncemented soil under both saturated and unsaturated conditions. The cementation bonding function is then combined with the unsaturated model by [27] to define a Cemented Unified Normal Compression Line (CUNCL) describing the virgin behaviour of both cemented and uncemented soils under unsaturated and saturated conditions.

\footnotetext{
* Corresponding author: agostino.bruno@,newcastle.ac.uk
} 
Overconsolidated states are described by assuming that the slope of a generic loading path tends asymptotically to the slope of the CUNCL as the soil state moves towards the CUNCL itself.

The complete model requires seven parameters, which have a clear physical meaning and are therefore relatively easy to calibrate. Three of these parameters govern the mechanical behaviour of the unsaturated uncemented soil under virgin conditions, which is here referred to as the "intrinsic behaviour" [28]. Additionally, two parameters describe the effect of cementation under virgin conditions, i.e. the "cemented behaviour". Finally, the remaining two parameters describe the progressive yielding and hysteretic behaviour of the overconsolidated soil.

The model has been calibrated and validated against isotropic tests on cemented soil samples under both saturated and unsaturated conditions [29]. Results show that the model can predict the main features of cemented, unsaturated soil behaviour such as the occurrence of gradual yielding and wetting-induced collapse. Further work is needed to couple the present mechanical law with a water retention law (similar to [30]) and incorporate non-isotropic stress states so that a complete hydromechanical framework for cemented unsaturated soils can be developed.

\section{Bounding surface mechanical model}

\subsection{Normally consolidated behaviour}

The model originates from the Unified Normal Compression Line (UNCL) [27], which describes the virgin variation of void ratio $e_{u}$ in uncemented soils under both saturated and unsaturated conditions in terms of a single stress variable named mean scaled stress $\bar{p}$ :

$$
\log e_{u}=-\lambda_{p} \log \frac{\bar{p}}{\bar{p}_{\text {ref }}}
$$

where $\lambda_{p}$ and $\bar{p}_{\text {ref }}$ are two model parameters representing the slope and intercept of the UNCL, respectively, in the $\log e_{u}-\log \bar{p}$ plane.

The mean scaled stress $\bar{p}$ is defined by [27] as the product of the mean average skeleton stress (also known as Bishop stress) $p^{\prime}$ and a power function of the degree of saturation $S_{r}$ :

$$
\bar{p}=S_{r}^{\frac{\lambda_{r}}{\lambda_{p}}} p^{\prime}
$$

where the mean average skeleton stress $p^{\prime}$ is defined as $p^{\prime}=p-u_{a}+S_{r} s$, where $s=u_{a}-u_{w}$ is the suction, $p$ is the mean total stress, $u_{a}$ is the pore air pressure and $u_{w}$ is the pore water pressure. The model parameter $\lambda_{r}$ defines the rate at which the porosity sustained by interparticle capillary bonds reduces as degree of saturation increases [27].

Note that the scaled stress $\bar{p}$ in Equation (2) becomes equal to the Terzaghi's effective stress when the degree of saturation is equal to one and, hence, the UNCL reduces to the normal compression line (NCL) of saturated soils.
In this paper, the UNCL is further extended to take into account the effect of cementation. To this end, the following cementation bonding function is introduced to describe the progressive reduction of the ratio between the cemented and uncemented void ratio $\frac{e}{e_{u}}$ (calculated at a given mean scaled stress) under virgin conditions, with increasing levels of mean scaled stress $\bar{p}$ :

$$
\frac{e}{e_{u}}=\left(\frac{R+\bar{p}}{\bar{p}}\right)^{\lambda_{c}}
$$

where the parameter $\lambda_{c}$ defines the rate at which interparticle linkages are broken with increasing stress levels while the parameter $R$ is the value of mean scaled stress corresponding to a ratio $\frac{e}{e_{u}}=2^{\lambda_{c}}$. An alternative expression of the cementation bonding function is obtained by applying logarithms to both sides of Equation (3), thus giving:

$$
\log \frac{e}{e_{u}}=-\lambda_{c} \log \left(\frac{\bar{p}}{R+\bar{p}}\right)
$$

which, after combination with Equation (1) leads to the following form of the CUNCL:

$$
\log e=-\lambda_{p} \log \left(\frac{\bar{p}^{\frac{\lambda_{c}}{\lambda_{p}}}}{\bar{p}} \bar{p}_{r e f}^{(R+\bar{p})^{\frac{\lambda_{c}}{\lambda_{p}}}}\right)
$$

The definition of "mean cemented scaled stress" $\overline{\bar{p}}$ as:

$$
\overline{\bar{p}}=\bar{p}\left(\frac{\bar{p}}{R+\bar{p}}\right)^{\frac{\lambda_{c}}{\lambda_{p}}}
$$

leads to a more compact expression of the CUNCL:

$$
\log e=-\lambda_{p} \log \left(\frac{\overline{\bar{p}}}{\overline{\bar{p}}_{\text {ref }}}\right)
$$

whose slope $\lambda_{p}$ in the $\log e_{u}-\log \overline{\bar{p}}$ plane is identical to the slope of the UNCL in the $\log e_{u}-\log \bar{p}$ plane. In Equation (7), $\overline{\bar{p}}_{\text {ref }}$ is the mean cemented scaled stress corresponding to a unitary void ratio, and therefore coincides with $\bar{p}_{\text {ref }}$, although the symbol $\overline{\bar{p}}_{\text {ref }}$ is used here for consistency with the mean cemented scaled stress.

The virgin behaviour of uncemented and cemented soils under both saturated and unsaturated conditions is therefore described by a total of five parameters (i.e. $\lambda_{p}, \lambda_{r} \overline{\bar{p}}_{\text {ref }}, R$ and $\lambda_{c}$ ).

The CUNCL expressed by Equation (7) delimits the region of overconsolidated soil states in the $\log e_{u}-$ $\log \overline{\bar{p}}$ plane. Inside this region, loading and unloading paths are defined by increasing and decreasing values of mean cemented scaled stress, respectively. The mathematical formulation of both loading and unloading paths is detailed in the following section.

\subsection{Overconsolidated behaviour}

Similar to [27], the model assumes that the slope of a generic loading path in the $\log e_{u}-\log \overline{\bar{p}}$ plane tends 
monotonically towards the slope of the CUNCL as this is approached, which is expressed as:

$$
\frac{d \log e}{d \log \overline{\bar{p}}}=-\lambda_{p}\left(\frac{\overline{\bar{p}}}{\overline{\bar{p}}}\right)^{\gamma}
$$

Equation (8) states that the derivative of the loading curve $\frac{d \log e}{d \log \bar{p}}$ is equal to the slope $\lambda_{p}$ of the CUNCL scaled by a factor smaller than one. The scaling factor is a power function of the ratio between the current value of mean cemented scaled stress $\overline{\bar{p}}$ and the image value $\overline{\bar{p}}_{i}$, while the exponent $\gamma$ is an additional model parameter. The image value of mean cemented scaled stress $\overline{\bar{p}}_{i}$ is obtained from the CUNCL corresponding to the current level of void ratio and is therefore obtained by inverting Equation (7) as:

$$
\overline{\bar{p}}_{i}=\frac{\overline{\bar{p}}_{r e f}}{e^{\frac{1}{\lambda p}}}
$$

Therefore, the ratio $\frac{\overline{\bar{p}}}{\overline{\bar{p}}_{i}}$ is always lower than one and tends to one as the loading path approaches the CUNCL.

By substituting Equation (9) into Equation (8), the following differential form is obtained:

$$
\frac{d \log e}{d \log \overline{\bar{p}}}=-\lambda_{p}\left(\frac{\overline{\bar{p}} e^{\frac{1}{\lambda_{p}}}}{\overline{\bar{p}}_{\text {ref }}}\right)^{\gamma}
$$

which can be integrated in a closed form, so that all loading paths are described by the following expression:

$$
e=\left[\left(\frac{\overline{\bar{p}}}{\overline{\bar{p}}_{\text {ref }}}\right)^{\gamma}+C_{L}\right]^{-\frac{\lambda_{p}}{\gamma}}
$$

The integration constant $C_{L}$ is unique to each loading path and is determined by imposing a suitable boundary condition, i.e. by introducing a known pair of values of void ratio and mean cemented scaled stress $\left(e_{0}, \overline{\bar{p}}_{0}\right)$ in Equation (11) as:

$$
C_{L}=e_{0}^{-\frac{\gamma}{\lambda_{p}}}-\left(\frac{\overline{\bar{p}}_{0}}{\overline{\bar{p}}_{\text {ref }}}\right)^{\gamma}
$$

During unloading, the derivative of the logarithm of void ratio with respect to the logarithm of mean cemented scaled stress is assumed constant and equal to $-\kappa$ as follows:

$$
\frac{d \log e}{d \log \overline{\bar{p}}}=-\kappa
$$

This assumption implies that all unloading paths are linear with identical slopes in the $\log e_{u}-\log \overline{\bar{p}}$ plane. It also means that the swelling index $\kappa$ is not affected by cementation and capillary bonding, whose effects are already incorporated in the definition of the cemented scaled stress.

Similar to loading paths, Equation (13) can be integrated in a closed form so that all unloading paths are described by the following expression:

$$
e=\frac{C_{U}}{\overline{\bar{p}}^{\kappa}}
$$

The integration constant $C_{U}$ is unique to each unloading path and is determined by imposing a suitable boundary condition, i.e. by introducing a known pair of values of void ratio and mean cemented scaled stress $\left(e_{0}, \overline{\bar{p}}_{0}\right)$ in Equation (14) as:

$$
C_{U}=e_{0} \overline{\bar{p}}_{0}^{\kappa}
$$

In summary, the complete model for overconsolidated soils requires seven material parameters:

a) three parameters $\left(\lambda_{p}, \lambda_{r}\right.$ and $\left.\overline{\bar{p}}_{\text {ref }}\right)$ describing the normally consolidated behaviour of the reference unsaturated soil (i.e. the intrinsic behaviour);

b) two parameters $\left(R\right.$ and $\left.\lambda_{c}\right)$ describing the effect of cementation on the normally consolidated behaviour of the reference unsaturated soil (i.e. the cemented behaviour);

c) finally, two additional parameters $(\gamma$ and $\kappa)$ describing the gradual yielding and the hysteretic behaviour of the overconsolidated soil.

Note that the intrinsic behaviour of the reference soil can be recovered by setting one of the two cemented parameters, i.e. $R$ or $\lambda_{c}$, to zero. This implies that the mean cemented scaled stress $\overline{\bar{p}}$ defined by Equation (6) reduces to the mean scaled stress $\bar{p}$ and hence the constitutive formulation reduces to that proposed by [27] for uncemented unsaturated soils.

The following sections present the calibration and the subsequent validation of the proposed model against the experimental data published by [29].

\section{Model calibration}

Model parameters were calibrated against two sets of isotropic loading-unloading tests on samples of compacted cemented sand [29]. Each set included two tests, namely one loading-unloading cycle under saturated conditions and one loading-unloading cycle under unsaturated conditions at a constant suction of $50 \mathrm{kPa}$ (Figures 1 and 2).

The samples of the first set of tests were prepared by mixing sand, filler and cement in the proportions of $96: 3: 1$, while in the second set of tests these proportions were varied to $96: 2: 2$. The percentage of fines (i.e. filler and cement) is therefore equal to $4 \%$ of the total mass for both sets of tests, although the average specific gravity $G_{s}$ is 2.65 for the first set and 2.64 for the second set, due to the slightly different proportions of cement and filler.

According to the nomenclature adopted by [29], each set is identified by the corresponding cement ratio $c$, which is the percentage of cement with respect to the total amount of fines (i.e. cement plus filler). The cement ratio $c$ is therefore $25 \%$ for the first set of tests and $50 \%$ for the second set of tests.

Samples were compacted under a static pressure comprised between 10 and $20 \mathrm{kPa}$ at a water content of $4 \%$ to attain a target dry density of $1380 \mathrm{~kg} / \mathrm{m}^{3}$. This resulted in a post-compaction void ratio of 0.92 for the first set of tests and of 0.91 for the second set of tests. 
The seven model parameters (i.e. $\lambda_{p}, \lambda_{r}, \overline{\bar{p}}_{r e f}, \gamma, \kappa, R$ and $\lambda_{c}$ ) were simultaneously calibrated by means of a multivariate best-fit of Equations (11) and (14) to the loading and unloading branches, respectively, of the tests from both sets. Identical values of the intrinsic and overconsolidated parameters (i.e. $\lambda_{p}, \lambda_{r}, \overline{\bar{p}}_{r e f}, \gamma$ and $\kappa$ ) were imposed for both sets of tests to reflect the similar nature of the reference soil. In contrast, the values of the cemented parameters (i.e. $R$ and $\lambda_{c}$ ) were allowed to vary between the two sets to account for the different cementation ratios (i.e. $\mathrm{c}=25 \%$ and $\mathrm{c}=50 \%$ ).

In each loading-unloading cycle, the integration constant $C_{L}$ of the first loading path was treated as an additional fitting parameter, while the integration constant $C_{U}$ of the subsequent unloading path was calculated using Equation (15) to ensure the continuity of the stress path at the reversal point between loading and unloading.

Table 1 summarises the values of all model parameters together with the integration constants $C_{L}$ (treated as independent fitting parameters) and $C_{U}$ (calculated by means of Equation (15)) of all loading and unloading paths performed on the two sets of samples (i.e. $c=25 \%$ and $\mathrm{c}=50 \%$ ) under both saturated and unsaturated conditions.

Note that experimental values of the degree of saturation were used for the calculation of the cemented scaled stress during the simulation of each test. Nevertheless, predicted values of degree of saturation could be equally used if the mechanical framework is coupled with a retention model similar to [30].

Figures 1 and 2 show the very good fit of the calibrated curves to the experimental data for both sets of tests, thus confirming the ability of the model to reproduce the variation of void ratio at different degrees of cementation under both saturated and unsaturated conditions. Note also that, for the saturated tests, the mean average skeleton stress reduces to Terzaghi's effective stress.

Table 1. Values of model parameters and integration constants

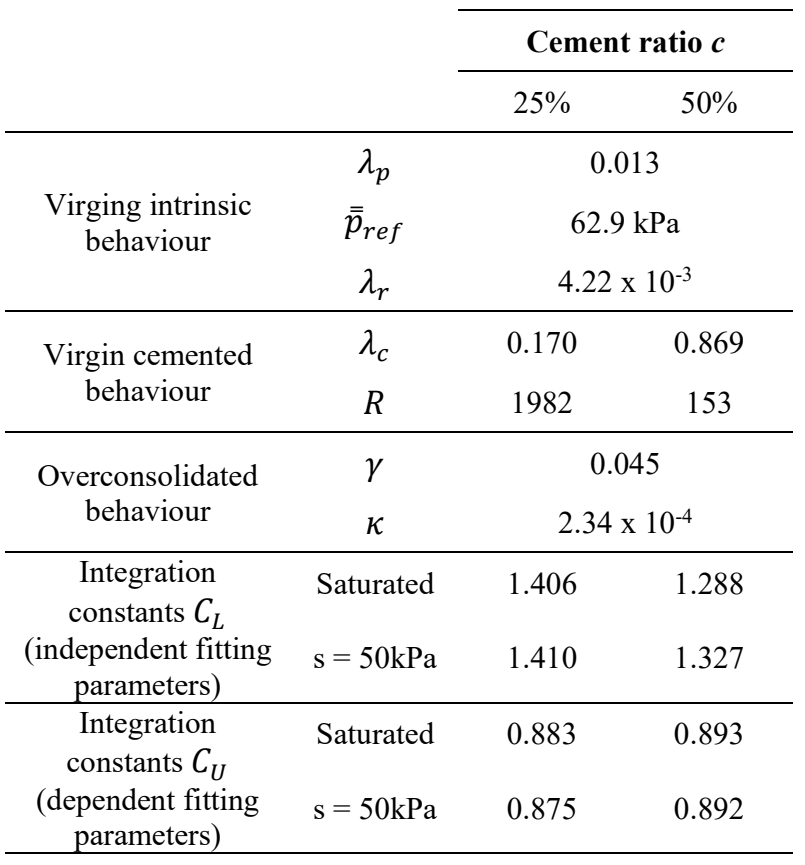

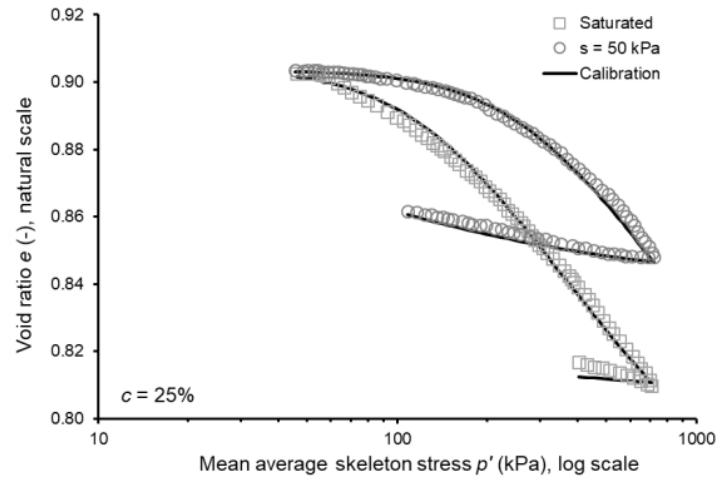

Fig. 1. Model calibration against saturated and unsaturated loading-unloading tests on samples with cement ratio $c=25 \%$ (the unsaturated test was performed at constant suction of 50 $\mathrm{kPa}$ ). Experimental data from [29].

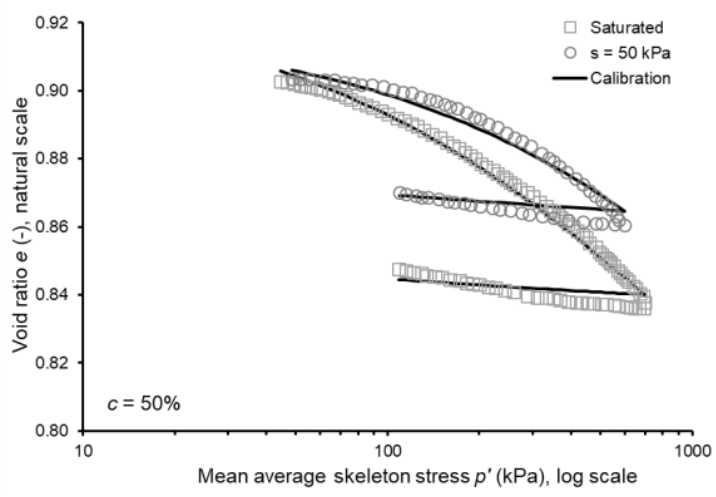

Fig. 2. Model calibration against saturated and unsaturated loading-unloading tests on samples with cement ratio $c=50 \%$ (the unsaturated test was performed at constant suction of 50 $\mathrm{kPa}$ ). Experimental data from [29].

\section{Model validation}

The capability of the proposed model to predict the mechanical behaviour of cemented unsaturated soils is assessed against additional tests which were not used in the calibration. In all simulations, the first integration constant was calculated by imposing that the experimental and predicted values coincide at the beginning of the test while the subsequent integration constant was calculated by imposing the continuity of the predicted curves at the transition between consecutive paths. As for the calibration stage, experimental values of the degree of saturation were used during the simulations for calculating the cemented scaled stress.

Figure 3 shows the experimental and predicted curves of a loading-wetting test on an unsaturated soil sample with a cement ratio $c$ of $25 \%$. The test consists of an isotropic loading path $\mathrm{AB}$, where the mean net stress increases from $40 \mathrm{kPa}$ to $600 \mathrm{kPa}$ at constant suction of $100 \mathrm{kPa}$, followed by a wetting path $\mathrm{BC}$, where the suction decreases from $100 \mathrm{kPa}$ to $5 \mathrm{kPa}$ at a constant mean net stress of $600 \mathrm{kPa}$. Inspection of Figure 3 indicates that the proposed model is able to capture both the gradual yielding during loading and the small collapse upon wetting.

Figure 4 shows the experimental and predicted curves of a loading-unloading test on an unsaturated soil sample 
with a cement ratio $c$ of $50 \%$. During the test, the mean net stress increases from $45 \mathrm{kPa}$ to $615 \mathrm{kPa}$ and then decreases back to $110 \mathrm{kPa}$ at a constant suction of $25 \mathrm{kPa}$. Even though the model predictions slightly underestimate the variation of void ratio during loading, the general trends of yielding upon loading and swelling upon unloading are reasonably well captured.

\section{Conclusions}

This paper has presented a bounding surface model capable of predicting the combined effect of cementation and partial saturation on the mechanical behaviour of soils subjected to isotropic stress states. The model is based on the definition of a cementation bonding function that relates cemented and uncemented void ratios under virgin
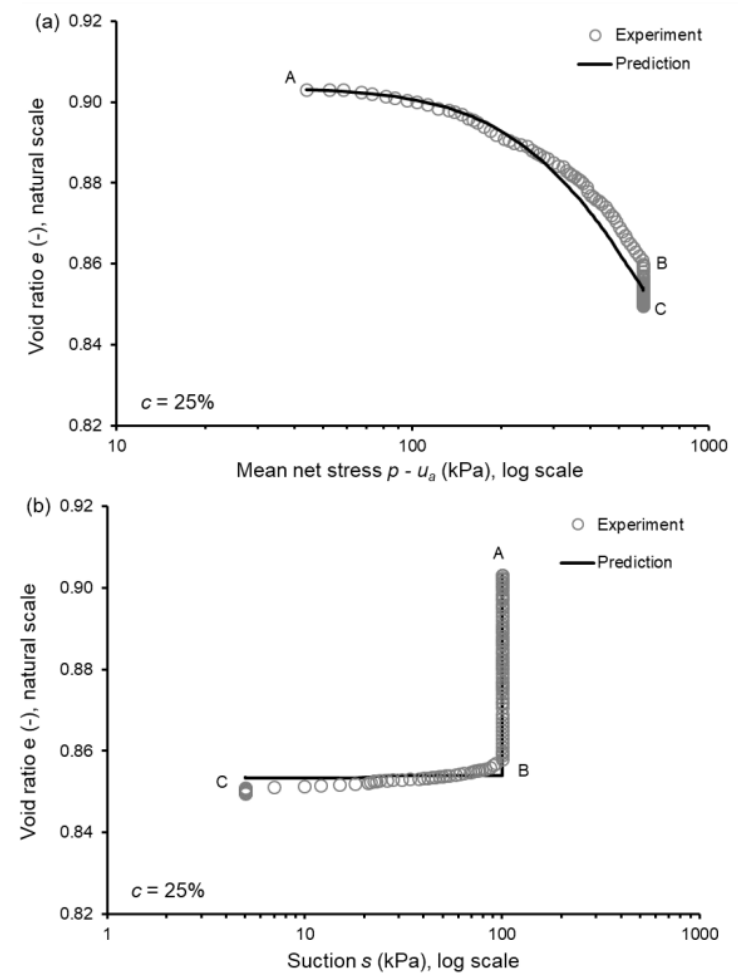

Fig. 3. Model prediction of unsaturated loading-wetting test on sample with cement ratio $c=25 \%$ : (a) void ratio vs mean net stress and (b) void ratio vs suction. Experimental data from [29].

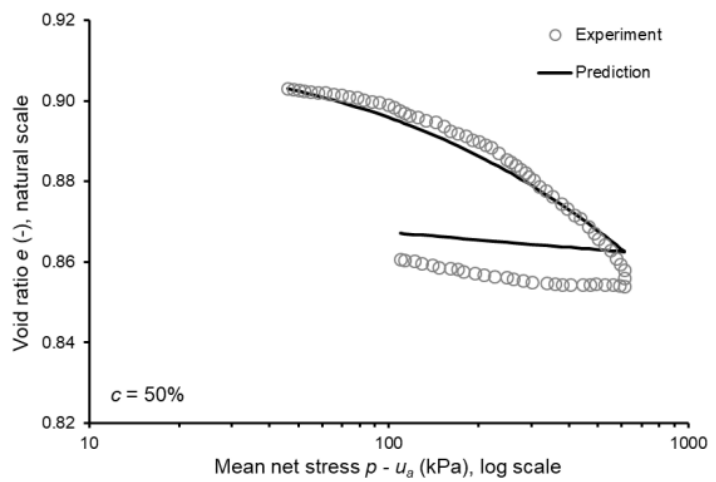

Fig. 4. Model prediction of unsaturated loading-unloading test on sample with cement ratio $c=50 \%$. Experimental data from [29]. conditions. According to this function, the extra porosity sustained by cementation reduces as inter-particle linkages are gradually broken under increasing stress.

The combination of the cementation bonding function with a previously proposed model for unsaturated soil behaviour has resulted in the definition of a cemented unified normal compression line (CUNCL) that predicts the virgin behaviour of both uncemented and cemented soils under saturated and unsaturated conditions. The CUNCL is expressed in terms of a single constitutive variable, named the mean cemented scaled stress which depends on both the mean average skeleton stress, and the degree of saturation. The mean cemented scaled stress reduces to Terzaghi's effective stress in the case of a saturated uncemented soil, i.e. when the degree of saturation becomes equal to one and at least one of the cemented parameters is zero.

The CUNCL defines a region of overconsolidated soil states, where loading and unloading paths are respectively defined by the increase and decrease of the mean cemented scaled stress. Gradual yielding is reproduced by assuming that the slope of the generic loading path tends to the slope of the CUNCL as the soil state approaches the CUNCL. Instead, during unloading, the model assumes a linear relationship between the logarithm of void ratio and the logarithm of the mean cemented scaled stress. Loading and unloading paths are described by two closedform equations that can also predict the hysteretic behaviour of soils during cycles of stress and/or suction. The model is formulated in terms of seven parameters describing the intrinsic behaviour of normally and overconsolidated soils, under both unsaturated and saturated conditions, and the effect of cementation on the behaviour of the normally consolidated soil.

The model performance has been validated against laboratory tests performed on cemented soil samples under saturated and unsaturated conditions. Comparison between experimental and predicted data has shown the model is able to capture the main features of cemented and unsaturated soil behaviour. Further work should be directed towards the extension of the present formulation to non-isotropic stress states and the incorporation of soilwater retention in order to produce a robust hydromechanical model for practical applications.

\section{References}

1. A. Gens, R. Nova, In Proceedings International Symposium on Geotechnical Engineering of Hard Soils-Soft Rocks, 485-494 (1993)

2. M. Kavvadas, A. Amorosi, Géotechnique, 50(3), 263-273 (2000)

3. M. D. Liu, J. P. Carter, Géotechnique, 50(4), 479-483 (2000)

4. M. Rouainia, D. Muir Wood, Géotechnique, 50(2), 153-164 (2000)

5. M. D. Liu, J. P. Carter, Can. Geotech. J., 39(6), 13131332 (2002)

6. R. Nova, R. Castellanza, C. Tamagnini, Int. J. Numer. Anal. Met., 27(9), 705-732 (2003) 
7. J. Vaunat, A. Gens, In Proceedings of the $12^{\text {th }}$ panamerican conference on soil mechanics and geotechnical engineering, 479-484 (2003)

8. B. Baudet, S. Stallebrass, Géotechnique, 54(4), 269278 (2004)

9. J. P. Carter, M. D. Liu, In Proceedings of the Geofrontiers congress - Soil constitutive models: Evaluation, selection, and calibration, 99-132 (2005)

10. M. D. Liu, S. Horpibulsuk, Y. J. Du, Geotech. Eng. J. Seags Agssea, 46(4), 96-101 (2015)

11. Z. Ali Rahman, D. G. Toll, D. Gallipoli, Geomech. Geoeng., 13(4), 233-245 (2018)

12. D. G. Fredlund, N. R. Morgenstern, Can. Geotech. J., 13(3), 261-276 (1976)

13. E. E. Alonso, A. Gens, A. Josa, Géotechnique, 40(3), 405-430 (1990)

14. S. J. Wheeler, V. Sivakumar, Géotechnique, 45(1), 35-53 (1995)

15. Y. J. Cui, P. Delage, Géotechnique, 46(2), 291-311 (1996)

16. D. A. Sun, H. Matsuoka, Y. Yao, W. Ichihara, Soils found., 40(3), 17-28 (2000)

17. B. Loret, N. Khalili, Mech. Mater., 34(2), 97-116 (2002)

18. D. Gallipoli, A. Gens, R. Sharma, J. Vaunat, Géotechnique, 53(1), 123-136 (2003)

19. S. J. Wheeler, R. S. Sharma, M. S. R. Buisson, Géotechnique, 53(1), 41-54 (2003)

20. M. Lloret-Cabot, S. J. Wheeler, M. Sanchez, Acta Geotech., 12(1), 1-21 (2017)

21. E. E. Alonso, A. Gens, Keynote Lecture in Proceedings of the $1^{\text {st }}$ International Symposium on Engineering Characteristics of Arid Soils, 173-205 (1994)

22. S. Leroueil, P. D. A. Barbosa, In Proceedings of the Asian Conference on Unsaturated Soils, 527-532 (2000)

23. B. Garitte, J. Vaunat, A. Gens, In Proceedings of the $4^{\text {th }}$ International Conference on Unsaturated Soils, 1944-1955 (2006)

24. P. N. M. Pinyol, J. Vaunat, J., E. A. P. de Agreda, Géotechnique, 57(2), 137-151 (2007)

25. C. Yang, Y. J. Cui, J. M. Pereira, M. S. Huang, Comput. Geotech., 35(6), 853-859 (2008)

26. J. M. Pereira, M. Rouainia, D. Manzanal, Journal of Geo-Engineering Sciences, 2(1-2), 3-16 (2014)

27. D. Gallipoli, A. W. Bruno, Géotechnique, 67(8), 703712 (2017)

28. J. B. Burland, Géotechnique, 40(3), 329-378 (1990)

29. L.C. Carvalho, PhD Thesis, UPC Barcelona (2002)

30. A. W. Bruno, D. Gallipoli, Comput Geotech, 110, 287-295 (2019) 